\title{
Other Medication
}

National Cancer Institute

\section{Source}

National Cancer Institute. Other Medication. NCI Thesaurus. Code C159287.

A directive to specify the other medication administered. 